\title{
Study on Strategies to Improve Tourist Satisfaction in Changbai Mountain Natural Reserve*
}

\author{
Mingju Liu \\ Yatai School of Business Administration \\ Jilin University of Finance and Economics \\ Changchun, China 130117
}

\author{
Liguang Zhao \\ School of Taxation \\ Jilin University of Finance and Economics \\ Changchun, China 130117
}

\author{
Yue Chen \\ Yatai School of Business Administration \\ Jilin University of Finance and Economics \\ Changchun, China 130117
}

\begin{abstract}
Taking Changbai mountain scenic area as an example, this paper proposes targeted countermeasures against the current satisfaction survey and existing problems which has provided feasible ideas and suggestions in order to improve the tourist satisfaction of Changbai mountain scenic area, improve the service quality of the scenic area, and develop a broader customer source market.
\end{abstract}

Keywords-Changbai Mountain natural reserve; tourist satisfaction; improvement strategy

\section{INTRODUCTION}

The proportion of tourism in the consumption structure of residents is increasing year by year, and the travel time is also concentrated on holidays, so there will be differences for tourist attractions in the peak season and the low season. The number of tourists in scenic spots has soared in the peak season, often saturated or even beyond the standard. If there is no effective strategy and plan in the scenic spot, it is easy to reduce the tourist satisfaction greatly, so as to affect the image of the scenic spot. Changbai Mountain Nature Reserve is one of the must-see attractions in Jilin Province, and it is a problem worth studying for how to improve the satisfaction of tourists in Changbai Mountain Nature Reserve and improve the service quality of scenic spots.

\section{OVERVIEW OF CHANGBAI MOUNTAIN NATURAL} RESERVE AND ITS DEVELOPMENT STATUS OF THE TOURISM

\section{A. Overview of Changbai Mountain Natural Reserve}

Changbai mountain natural reserve is the birthplace and a sacred mountain of Manchu in China, one of the ten famous mountains in China. It has been recorded as early as 4,000 years ago: The Classic of Mountains and Seas calls it as "unsalted mountain". In the northern Wei dynasty, it was

*Fund Project: The project of the National Natural Science Foundation of China (Project No.: 41401146); Jilin Province Science and Technology Development Plan Project (20150204040NY); Jilin Provincial Department of Education Project (JJKH20170140SK). called "Tumountain", in the tang dynasty, "Taibai Mountain", and in the Jin dynasty, "Changbai Mountain". During the Qing dynasty, it was regarded as a sacred mountain, and the emperor had to personally or appoint his ministers to worship it in Jilin Wula (now Jilin).The general peak is the highest peak of Changbai mountain, with a peak of 2,750 meters. Famous scenic spots include the hot spring square, the magnificent waterfall, the mysterious and changeable Tianchi, the small jade-like silver fish lake, the jade-green deep lake, the quiet and deep underground forest. It belongs to moderate continental mountain climate, with climate changing vertically. Natural resources, plants and animals are abundant and diverse. It is known as the "natural museum" for its 50 species of animals, 1,800 species of higher plants, 280 species of birds, 50 species of fish and 1000 species of insects.

\section{B. Tourism Development Status of Changbai Mountain Natural Reserve}

Changbai Mountain, one of the top ten famous mountains in China, attracts tourists from all over the country every year. With the improvement of the national standard of living, the proportion of tourism in household consumption expenditure also increases year by year. According to statistics, in 2017, the whole scenic area received 2.23 million persons, an increase of $2.2 \%$ over the previous year, and had tourism revenue of 600 million yuan, an increase of $2.6 \%$ over the previous year. Now living in cities, people have a fast living space and a big pressure. Every day, they face concrete structures, feeling no closer to nature. As a result, among the options for the holiday, people are more inclined to choose go close to the nature. As a natural "oxygen bar" and "natural museum", Changbai Mountain will become a hot spot in the future tourism industry. 


\section{PROBLEMS IN TOURISM DEVELOPMENT OF CHANGBAI MOUNTAIN NATURAL RESERVE}

\section{A. Long Waiting Time for Tickets}

With the development of the Internet, online ticket purchasing has become a more convenient way, but the traditional window ticket purchasing still occupies a large proportion. Due to the limited ticket windows, during the holiday season, the waiting time for tickets is bound to increase substantially. Usually at the peak of the peak tourist season, it takes up to two hours for tourists to wait in line outside the scenic area. What's more, after the purchase, it usually takes 15 minutes to half an hour to check in and wait for the bus. Long waiting in line will greatly reduce tourists' satisfaction with the scenic area, reduce the rate of revisiting, affect tourists' overall evaluation of the scenic area, and then affect the long-term survival and development of the scenic area. Therefore, reducing the waiting time is an important problem to be solved urgently.

\section{B. The Traffic Management within the Scenic Area Is Not Standardized}

There are 85 coaches and 28 midibuses in Changbai scenic area. The bus mainly transports tourists from the mountain gate to the waterfall in the scenic area and stops at all the scenic spots during the return period. There is no fixed stopping time. Minibuses are mainly responsible for transporting tourists between various scenic spots. When there are a small number of tourists, the traffic in the scenic area can fully meet the requirements of tourists and service requirements. However, during the peak season, a large number of tourists are often stranded at the traffic hub of the scenic spot, unable to get on a bus. Also, there are no special passageways or instructions for tourists to queue up for the ride. Usually, when the bus stops, the tourists rush in, and it has caused the order chaotic. It is easy to have accidents and thus it threatens the safety of tourists. Secondly, the traffic dispatch management is not standardized, and there is no unified allocation plan and allocation principle. It often happens that a large number of tourists are stranding at the bus stops while there are empty cars passing by.

\section{Unreasonable Sightseeing Routes}

During the National Day and Mid-Autumn holidays in 2017, the Changbai mountain scenic area received a total of 181,400 tourists, with an increase of $23.74 \%$ year on year. Among them, the west scenic area received 60,300 tourists, with an increase of 13,300 from last year's 47,000, an increase of $28.3 \%$ over the same period last year. The north scenic area received 121,100 tourists, with an increase up to 21,500 from last year's 99,600 and a year-on-year increase of $21.59 \%$. On October 4 , the Changbai mountain scenic area received a total of 36,200 tourists in a single day, which reached the peak of large reception. Faced with the increasing number of tourists, the scenic spots still follow the usual sightseeing plan on the tourist route. It has no effective guide for tourists to avoid the peak season or re-plan the tour route. However, under the condition of tourists' congestion, tourists are forced to be separated, which leads to the dissatisfaction of tourists during the tour and affects their overall evaluation of the scenic spot. The revisiting rate of tourists to Changbai Mountain is greatly reduced, which is not conducive to the long-term development of the scenic area.

\section{ANALYSIS OF FACTORS INFLUENCING TOURISTS' SATISFACTION}

\section{A. The Environment and Infrastructure of the Scenic Area}

The environment and infrastructure of scenic spots are the most basic conditions to determine tourists' satisfaction. As a tourist destination, the attraction of the environment of scenic spots is not only due to the resource conditions of the tourist destination, but also to the subsequent development, construction and management. Well-developed, innovative and humanistic tourism destinations will naturally attract tourists. After having the innate environmental advantages, the subsequent management construction is very important. The signs and knowledge signs in the scenic area, the sanitary environment, the establishment of accommodation, catering, entertainment, rest, participatory projects, signage signs, safety signs, etc., all of these amenities, as well as some humane and subtle details, can have a positive impact on improving visitors' satisfaction. Second, to standardize supporting facilities, to guarantee the rest areas clean, for example, to make the public toilet hygienic, to have clear guide interpretation, unified work clothes for scenic spot workers. Third is to have certain humanistic characteristics. For example, buildings with local characteristics are built in the scenic area, certain cultural features are given to accommodation, restaurants and rest areas in the scenic area, and folk activities with local characteristics are organized for tourists to participate. In turn, the visiting time of tourists is extended to improve their satisfaction.

\section{B. Convenient Transportation}

The convenience of transportation sometimes decides whether tourists choose a scenic spot as their destination, so transportation is one of the prerequisites for the development of tourism. According to a survey, the time spent on transportation takes up the majority of the time spent on traveling. The fatigue caused by the long journey will increase the fatigue of the tourists, which will affect the tourists' interest and their satisfaction of the scenic spots. Therefore, the requirements of accessibility, convenience, safety, comfort and sightseeing of the traffic are all important in the management of scenic spots.

\section{Service Quality of Catering and Shopping}

The purposes of travels are not only to enjoy the scenery and culture, but also to enjoy local delicacies and shopping. Therefore, it is especially important to improve the service quality of catering and shopping. As a culture, diet shows the characteristics of a region, and the connotation of historical inheritance is the most direct way to understand a place. Usually, the shopping behavior of tourists in the scenic area is mostly to buy gifts to others. Therefore, high-volume shopping experiences and high-quality tourism products are also a form of publicity for tourism destinations. It has the most direct 
effect on improving tourists' satisfaction. Huang Jing et al. found that tourist destinations are direct consumption places of tourism products and services, so they studied the impact of various services provided by tourist destinations on tourists' satisfaction. The tourism environment of the destination, basic and non-basic tourism service supply, integrated tourism service supply and other 7 aspects have a major impact on tourists' satisfaction.

\section{Large Differences from Expectations}

Expectation is the hope and expectation of something or someone. Visitors' expectation refers to the psychological expectations that tourists get through their own processing of some evaluation and information about the destination before going to visit. Therefore, if tourists conduct tourism activities, they will find that the actual situation of the tourist destination is higher than their expectations, and they will feel satisfied. When the tourist destination is lower than what they expected or even far lower, tourists tend to resist and complain in the tour, which directly leads to the decrease of their satisfaction to the tourist destination. On the one hand, the reason for the great disappointment in tourists' expectations may be the exaggerated publicity of tourist destinations, which describes a perfect image of tourist destination for tourists that is quite different from the actual landscape. Although it will increase the attraction of tourist destinations in the short term after such promotion, the revisiting rate of tourist destinations will be greatly reduced. On the other hand, everyone has different views of appreciation. Tourists come because of the recommendation of others. Josip Mikulic measured the impact of tourists' expectations on the satisfaction of Malaysia, Korea, Japan and tourists from other countries in Australia. Akin points out that tourist satisfaction influenced by expectations is often crucial for a successful marketing, destination choice, consumer products and services, and tourists' decision to revisit.

\section{STRATEGIES TO IMPROVE TOURISTS' SATISFACTION}

\section{A. Improve Infrastructure Construction and Build Convenient Supporting Facilities}

The main feature of Changbai mountain scenic area is its original ecological natural landscape, with few activities that tourists can participate in. Tourists spend most of their day in the scenic area walking outdoors, so they spend more time physically. Therefore, more places should be set up for tourists to rest. Meanwhile, it also provides catering services to meet the needs of tourists to rest and eat. What's more, architecture and food should be able to display local characteristics to attract tourists. Second is the comprehensive coverage of the network. In today's Internet era, people are transmitting information through the Internet all the time, especially young people, who are willing to share their lives with the outside world in the form of live broadcast. Therefore, the coverage of the network allows tourists to broadcast the landscape of the tourist destination, which is also a kind of publicity and marketing for the tourist destination, which is more attractive than tourism promotion and can save costs.

\section{B. To Increase the Number of Traffic Shifts and to Improve the Scheduling Efficiency}

During the peak period of the peak season of scenic spots, most of the ways to reach the scenic spot are taking by bus and the number of tourists is more than 12,000 .How to take into account the convenience of tourists and the safety of the tour process, and how to reduce the phenomenon of tourists' long stay are the important problems to be solved at the present stage. First, the number of transport vehicles in the scenic area will be increased, the number of transport shifts will be increased, and the number of tourists will be increased during the same period. At the same time, the concept of "landscape transportation" should be integrated, so that the passenger transportation is not a single function of delivery, but also another kind of "rest tour". The two sides of the transport line should have some special scenery to make the journey more interesting. Waiting for a long period of time will not only shorten tourists' time for other attractions but will also cause them to complain a lot and reduce their satisfaction. At the same time, they will produce garbage in a waiting area, increasing the burden of the staff of the scenic spot. The frequency of scheduling should be increased so that tourists' sightseeing activities can be well connected, which makes tourists spend more time on visiting scenic spots rather than waiting.

\section{To Ensure Food and Beverage to Be Safe and Hygienic to Improve Service Quality}

Tourism products cover all aspects of food, housing, transportation, travel, shopping and entertainment. Among them, food accounts for a high proportion of tourists' consumption. Therefore, the emphasis on food safety is particularly important. In terms of food safety, first of all, we should strengthen the supervision and control of food safety. All food products in the scenic area and special products sold to tourists should be strictly controlled and tracked to ensure that the food source channel and processing process are safe and hygienic.

As a service industry, tourists' satisfaction comes from their perception of the overall service process. Only with high service quality can tourists get high praise. Scenic area can improve the service quality from the small work like the specified language of the stuff, guide services, public order, health, clear and distinctive attractions such signs. For example, the guide's explanation should be clear, and the content should be reasonable, so that tourists can not only appreciate the beautiful scenery, but also understand all the cultural and geographical knowledge contained in each scenic spot. Volunteer interpreters can also be openly recruited. Usually the facilitator is a local resident who has studied the human geography of the Changbai Mountain and has been living at the foot of the Changbai Mountain for all the time. They know everything about climate changes and every kind of plants. It adds interesting and personalized features to the process of tourists.

\section{Combination of Natural Landscape and Culture}

A single natural landscape will make the tour boring. Therefore, the combination of nature and humanity can also 
increase the participation of tourists. Only a perfect tour can give tourists a high degree of satisfaction. Changbai mountain scenic area mainly consists of natural landscape, with a complex topography and landform, diverse animal and plant species, and rich mineral resources. By virtue of its unique advantages, the scenic spot can explain geographical knowledge to tourists on site to make them better understand the great rivers and mountains of the motherland. Therefore, tourists can learn knowledge while play. The author thinks that this can attract more teenagers and scholars in related fields. You can even work with schools and colleges to host summer camp teams. Therefore, the fun of the tour process is raised, so as to obtain higher tourist satisfaction.

\section{E. To Innovate Publicity Methods and Improve Tourism Image}

Tourism image is the direct condition to attract tourists to carry out tourism activities, and it is also an important index to influence tourists' satisfaction. In the eyes of tourists, the image of Changbai mountain scenic area is relatively low. The reason is that except the northeast parts of tourists, the rest of the group know a little about Changbai Mountain, or even nothing at all. It shows that the scenic area is not proper in publicity or not does enough to advertise, not being successful in attracting tourists. First of all, we should make a positioning for the scenic area and make a propaganda slogan. The positioning of the scenic area determines its focus of the tourists group and the expression of its characteristics. In the publicity, the features of Changbai mountain scenic area should be fully displayed and the publicity channels should be diversified. Now the Internet is highly developed and it is an era of information. Therefore, we should make full use of the influence of the Internet in publicity. At the same time, the propaganda should not be exaggerated, which will lead to excessive psychological expectations of tourists. If the actual situation is lower than tourists' psychological expectations after arriving at the scenic spot, there will be complaints, which will directly lead to the decrease of their satisfaction. Therefore, the publicity must proceed from the actual situation, carry out targeted publicity, and do not over-process the image of the tourist destination. In this way, they can make tourists' psychological expectations reach a reasonable level.

\section{F. To Realize "Smart Tourism" with Big Data}

The concept of "smart tourism" was proposed in 2010, and the national public service network and platform of smart tourism were established in 2016. It is expected to build a systematic value chain network of smart tourism in Jianshi county by 2020 . In the Internet era of big data, promoting the "wisdom tourism" can enable the visitors to get a real-time understanding of tourism information, make the tourism planning, at the same time help the spots to alleviate the pressure of the peak season. Let the wireless network covers the whole attractions and scenic spots to allow visitors to inquire and receives the real-time number tourists in tour spots nearby to decide whether they need to plan tour routes to avoid crowded time. The tour routes of Changbai mountain usually follow the tour of waterfall, hot spring square, small Tianchi, green deep pool, Tianchi and underground forest. At the same time, the transportation line also first carries guests to the waterfall spa plaza, which usually caused the hot spring square to be crowded with tourists while the underground forest scenic spot below the mountain is empty. Through the introduction of the concept of the "wisdom tourism", it can make people spread out to various attractions, alleviate traffic congestion phenomenon, and provide visitors with a more comfortable environment, so as to improve the satisfaction of tourists.

\section{CONCLUSION}

This paper discusses the recent status of Changbai mountain natural reserve and the improvement of tourist satisfaction and puts forward some practical suggestions. First of all, the scenic area should set out from them, find out the problems existing in the management process of scenic spots, and excavate the advantages of Changbai mountain natural reserve, making it a competitive advantage in the tourism market. Secondly, the factors influencing tourists' satisfaction are analyzed. From the perspective of tourists themselves, the factors include their perceived value, psychological expectation and motivation of tourism. From the view of the scenic area, the factors include its tourism resources, tourism image and service quality. Finally, the tourist satisfaction strategy of Changbai mountain natural reserve is proposed. It can be used for reference in the future development of Changbai mountain scenic area to find its advantages and positioning.

\section{REFERENCES}

[1] Zhai Dongfang. Evaluation and improvement strategy of urban tourist satisfaction in Henan province [D] Zhengzhou: Zhengzhou university, 2013

[2] Liu Mingju et al. Study on the sustainable development of tourism in north Changbai mountain scenic area [J]. Tax and economy, 2015 (5)

[3] Liu Mingju et al. Study on the current situation and countermeasures of tourism development in the northern scenic area of Changbai mountain [J]. Green technology, 2015(1)

[4] Liu Mingju, yuan fang. Some thoughts on tourism resources research [J]. Tax and economy, 2016 (1)

[5] Li Guang. Study on forest tourism value of north slope of Changbai mountain [J]. Natural science, 2002. (2)

[6] Huang Jing et al. Analysis on the impact of tourism destination supply chain management on tourists' satisfaction [J]. Journal of tourism, 2010. (8)

[7] Fu Quansheng. Theory and measurement of satisfaction of protected tourists [J]. Industry and market, 2005. (5)

[8] Yang Lichun et al. Study on countermeasures to optimize the management of China's tourist attractions [J]. Foreign trade and economic cooperation, 2015. (10)

[9] Josip Mikulic.Darko Prebezac.Using dummy regression to explore asymmetric effect in tourist satisfaction:A cautionary note [J].Tourism Management,2016(5)

[10] Akin Aksu, Ebru Tarcancigen.RuyaEhtiyar,A Comparison of Tourist Expectation and Satisfaction:A Case Study from Antalya Region of Turkey [J].TURIZAM,2010,14(2)

[11] Jia Jianxi. On customer's satisfaction of tourism products [J]. Economic experience, 2014. (1)

[12] Qian Linlin. Research on traffic organization and management methods of large tourist attractions [J]. Application of transport tools,2015. (2)

[13] Wang Tianying. Research on the development status and countermeasures of intelligent tourism in Hebei province [J].2015. (9). 